56

57

58

59

Revista Colombiana de Educación

Aporte de Piaget al campo de la Educación y la Pedagogía 
Revista Colombiana de Educación. --N. ${ }^{\circ} 1$

(Ene. -jun., 1978)_. -- Bogotá, Colombia :

UPN.DGP.CIUP, 1978.

Semestral

ISSN 0120-3916

1. Educación - Publicaciones Periódicas. 2. Colombia - Educación. 3 Investigación Educativa. I. Universidad Pedagógica Nacional. División de Gestión de Proyectos. Centro de Investigaciones.

Revista indexada por Colciencias en categoría B y por los índices internacionales: Internacional Bibliography of the Social Sciences (IBSS);

Educational Research Abstracts (ERA);

Índice de Revistas de Educación Superior e Investigación Educativa (IRESIE);

Sistema Regional de información en línea para Revista Científicas de América Latina, el Caribe, España y Portugal (LATINDEX),

Biblioteca Digital OEl; Ulrich Periodicals Directory;

Handbook of Latinamerican Studies of Library of Congress.

DIALNET

http://www.pedagogica.edu.co/revistas/ojs/index.php/RCE

ISSN 0120-3916

Copyright:

Los artículos de este número se pueden utilizar,

siempre y cuando se cite la fuente.

Tarifa Postal reducida por Resolución 2244

de septiembre de 1990 de la Administración Postal

División Gestión de Proyectos, Centro de Investigaciones

Universidad Pedagógica Nacional, DGP-CIUP.

Av. Calle 127 N. ${ }^{\circ} 11-20$, Bogotá, D. C. Colombia

Datos de contacto: (057-1) 6156537 - 6261048

rce@pedagogica.edu.co

rceupn@gmail.com

Responsable del canje:

Miriam Aixa González

División de Biblioteca y Recursos Bibliográficos

mgonzale@pedagogica.edu.co

Teléfono (057-1) 5941894, ext. 175

Suscripción Nacional e Internacional.

Impresión: Invergraficas R y M Sas

Bogotá, 2011 


\section{Revista Colombiana de Educación}

Aporte de Piaget al campo de la Educación y la Pedagogía

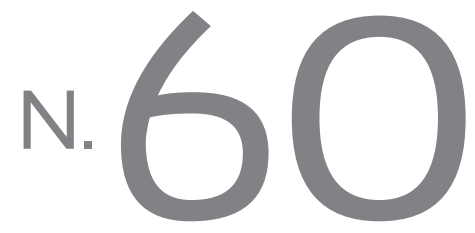

Bogotá - Colombia

Primer semestre de 2011

\section{Rector}

Juan Carlos Orozco Cruz

Vicerrector Académico

Edgar Alberto Mendoza Parada

Vicerrector de Gestión Universitaria

José Domingo Garzón Garzón

Director de la Revista y del Centro

de Investigaciones -CIUP-

Gustavo Castillo

\section{Editor Temático}

Christian Hederich Martínez

Asistente Editorial y Corrección de estilo

Lucía Bernal Cerquera

Monitor

Jerson Hernández de la Cruz

Preparación editorial

Universidad Pedagógica Nacional

Fondo Editorial

Coordinador

Luis Emilio Ávila Rodriguez

Rediseño y Diagramación

Diseño de caratula

Juan Camilo Corredor Cardona 
La Revista Colombiana de Educación es una publicación semestral del Centro de Investigaciones de la Universidad Pedagógica Nacional que data de 1978, y que ha pretendido ser una publicación de referencia para los estudiosos de la Educación y la Pedagogía. El público al que se dirige la revista contempla a los especialistas en temáticas educativas y pedagógicas, al magisterio en general y a estudiantes universitarios de pregrado y posgrado de las áreas de Educación, Ciencias Sociales y Humanidades.

La responsabilidad de las opiniones que se exponen en los artículos corresponde a sus autores.

\section{Comité Editorial RCE}

\begin{tabular}{|c|c|c|}
\hline Nombre & Afiliación & Correo \\
\hline Alberto Martínez Boom & $\begin{array}{l}\text { Universidad Pedagógica } \\
\text { Nacional, Bogotá, Colombia }\end{array}$ & almarboom@yahoo.com \\
\hline Martha Cecilia Herrera & $\begin{array}{l}\text { Universidad Pedagógica } \\
\text { Nacional, Bogotá, Colombia }\end{array}$ & malaquita10@gmail.com \\
\hline Adolfo León Atehortúa Cruz & $\begin{array}{l}\text { Universidad Pedagógica } \\
\text { Nacional, Bogotá, Colombia }\end{array}$ & adolfoatehortua@cable.net.co \\
\hline Christian Hederich Martínez & $\begin{array}{l}\text { Universidad Pedagógica } \\
\text { Nacional, Bogotá, Colombia }\end{array}$ & christian.hederich@gmail.com \\
\hline Rosalba Pulido de Castellanos & $\begin{array}{l}\text { Universidad Pedagógica } \\
\text { Nacional, Bogotá, Colombia }\end{array}$ & rpulido@pedagogica.edu.co \\
\hline Emilio Tenti Fanfani & $\begin{array}{l}\text { Instituto Internacional } \\
\text { de Planeamiento de la } \\
\text { Educación IIPE, Unesco } \\
\text { (Argentina) }\end{array}$ & emilio@iipe-buenosaires.org.ar \\
\hline Mariano Narodowki & $\begin{array}{l}\text { Universidad Torcuato Di } \\
\text { Tella (Argentina) }\end{array}$ & mnarodowski@utdt.edu \\
\hline Gabriela Diker & $\begin{array}{l}\text { Centro de Estudios Multidi- } \\
\text { ciplinarios (Argentina) }\end{array}$ & gdiker@fibertel.com.ar \\
\hline Aimer Granados & $\begin{array}{l}\text { Universidad Autónoma } \\
\text { Metropolitana (México) }\end{array}$ & aimer@correo.xoc.uam.mx \\
\hline Olga Lucía Hoyos de los Rios & $\begin{array}{l}\text { Universidad del Norte, } \\
\text { Barranquilla, Colombia }\end{array}$ & ohoyos@uninorte.edu.co \\
\hline Agueda Bittencourt & $\begin{array}{l}\text { Universidad de Campinas } \\
\text { (Brasil) }\end{array}$ & agueda@unicamp.br \\
\hline \multicolumn{3}{|l|}{ Comité Científico } \\
\hline Gabriela Ossenbach & España & gossenbach@edu.uned.es \\
\hline Pablo Pineau & Argentina & pablopineau@gmail.com \\
\hline Rene Rickenmann & Suiza & Rene.Rickenmann@unige.ch \\
\hline Santiago Estaun & España & Santiago.Estaun@uab.cat \\
\hline Eveline Algebaile & Brasil & ealgebaile@gmail.com \\
\hline Maria do Carmo Martins & Brasil & carminhapousa@gmail.com \\
\hline
\end{tabular}




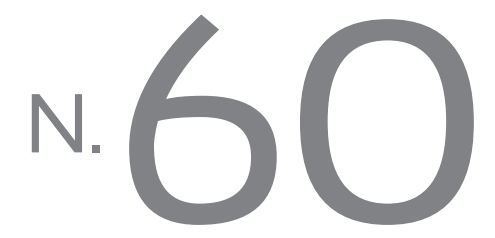

\section{Arbitraje}

Teresa León Pereira. Asociación Colombiana para el Avance de la Ciencia, ACAC.

Asociación de profesionales ProCalidad de la Educación, ASPROED. telepereira@yahoo.com Liliana Chaves Castaño. Universidad de Antioquia y CINDE. clasesliliana@gmail.com

Miguel Chajin Flórez. Docente investigador. Universidad Autónoma del Caribe. mchajin@hotmail.com

Gloria Patricia Marciales Vivas. Docente. Pontificia Universidad Javeriana gloria.marciales@ javeriana.edu.co

Ángela Camargo Uribe. Docente, Investigadora. Universidad Pedagógica Nacional. acamargo@yahoo.es

Ligia Inés García C. Docente e investigadora departamento de Educación y Maestría en Enseñanza de las ciencias. Universidad Autónoma de Manizales. ligarcia@autonoma.edu.co, ligiaines.garcia@gmail.com
Christian Hederich Martínez. Docente investigador. Universidad Pedagógica Nacional. christian.hederich@gmail.com

Ara Mercedes Cerquera Córdoba. Docente Asociada, Investigadora. Universidad Pontificia Bolivariana. Bucaramanga. aramercedes@yahoo.com

Sandra Patricia Peña Bernate. Docente. Universidad de Ibagué. sandra.bernate@ unibague .edu.co

Nohelia Hewitt Ramírez. Decana Facultad de Psicología. Universidad de San Buenaventura Sede Bogotá. nhewitt@usbbog. edu.co

Edward Leonel Prada Sarmiento. Docente Asociado Investigador. Universidad Pontificia Bolivariana. Bucaramanga. edward.prada@upb.edu.co 


\section{Contenido}

Reflexiones

15

La presencia de Piaget en la

Educación colombiana, 19602010

\section{Carlos Eduardo Vasco}

$15-40$

El legado de Piaget a la

Didáctica de la geometría

Leonor Camargo Uribe

41-60

Implicaciones de la teoría

piagetiana en la enseñanza de las Ciencias Sociales

Sandra Marcela Ríos

Juan Carlos Ramos

61-70
Piaget: lenguaje, conocimiento y Educación

Alfonso Cárdenas Páez

71-91

Aporte de la obra de Piaget a la comprensión de problemas educativos: su posible explicación del aprendizaje

Nicolás Arias Velandia

Rita Flórez Romero

93-105

De la Epistemología constructivista piagetiana, el reconocimiento de la cultura y de la diversidad para la formación en escenarios culturalmente diversos

Norma Constanza Castaño

107-122 


\title{
Revista Colombiana de Educación
}

\author{
Enero-julio 2011 edición 60
}

La tardía instalación de la teoría del desarrollo cognitivo de Jean Piaget en Colombia, 1968 - 2006

Absalón Jiménez

$123-140$

Presencia del espíritu

piagetiano en los lineamientos de política educativa en Bogotá

Carlos Alberto Abril

$141-150$

Piaget y Bourdieu: elementos teóricos para una pedagogía ciudadana

Álvaro Moreno Durán

Faustino Peña Rodríguez

152-162
Reseñas

163

Reseña de la tesis doctoral

"Aprendizaje autorregulado, estilo cognitivo y logro académico en ambientes computacionales". Autoría, Omar López Vargas

\section{Christian Hederich Martínez}

$165-172$

Reseña de la tesis "Dimensiones interactiva, discursiva y didáctica del estilo de enseñanza. El caso de las ciencias naturales". Autoría, Ángela Camargo

Christian Hederich Martínez 173-177 


\section{Content}

Reflections

15

Piaget's Presence in Colombian

Education, 1960-2010

Carlos Eduardo Vasco

$15-40$

Piaget's Legacy for Geometry

Teaching

Leonor Camargo Uribe

41-60

Repercussions of Piaget's Theory on Social Science

Education

Sandra Marcela Ríos

Juan Carlos Ramos

61-70
Piaget: Language, Knowledge and Education

Alfonso Cárdenas Páez

71-91

A Contribution by Piaget's Work to Understanding Educational Issues: A Probable Learning Explanation

Nicolás Arias Velandia

Rita Flórez Romero

93-105

Piaget's Constructivist

Epistemology, Recognition of Culture and Diversity for Training in Culturally Dissimilar Environments

Norma Constanza Castaño

107-122 


\title{
Revista Colombiana de Educación
}

\author{
Enero-julio 2011 edición 60
}

Belated Implementation in Colombia of Jean Piaget's Theory of Cognitive Development, 1968-2006

Absalón Jiménez

$123-140$

Presence of Piegetian Spirit in the Educational Policy Principles in Bogotá

Carlos Alberto Abril

$141-150$

Piaget and Bourdieu: Some Theoretical Elements for a Public Pedagogy

Álvaro Moreno Durán

Faustino Peña Rodríguez

152-162

\section{Reviews}

163

Doctorate Thesis Review "Self Regulated Learning, Cognitive Style and Academic Success in Computational Environments" by Omar López Vargas

\section{Christian Hederich Martínez}

165-172

Doctorate Thesis Review "Learning Style: Interactive, Discursive, and Didactical Dimensions, Case of Biological Science" by Ángela Camargo Christian Hederich Martínez 173-177 